\title{
Anxiety and depression in Dutch patients with primary ciliary dyskinesia and their caregivers: associations with health-related quality of life
}

\author{
Marieke Verkleij ${ }^{1}$, Iris Appelman ${ }^{1}$, Josje Altenburg ${ }^{2}$, Jos Twisk ${ }^{3}$, Alexandra L. Quittner ${ }^{4}$ and Eric Haarman ${ }^{5}$
}

${ }^{1}$ Dept of Pediatric Psychology, Amsterdam UMC, Vrije Universiteit Amsterdam, Amsterdam, the Netherlands. ${ }^{2}$ Dept of Pulmonology, Amsterdam UMC, Amsterdam, the Netherlands. ${ }^{3}$ Dept of Epidemiology and Data Science, Amsterdam UMC, Amsterdam, the Netherlands. ${ }^{4}$ Behavioral Health Systems Research, Miami, FL, USA. ${ }^{5}$ Dept of Pediatric Pulmonology, Emma Children's Hospital, Amsterdam UMC, Vrije Universiteit Amsterdam, Amsterdam, the Netherlands.

Corresponding author: Marieke Verkleij (m.verkleij@amsterdamumc.nl)

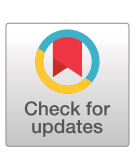

This version is distributed under the terms of the Creative Commons Attribution Non-Commercial Licence 4.0. For commercial reproduction rights and permissions contact permissions@ersnet.org

Received: 20 April 2021 Accepted: 2 July 2021

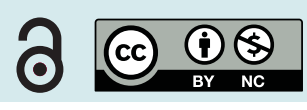

\section{Shareable abstract (@ERSpublications)}

Individuals with PCD and their caregivers are at risk of developing symptoms of anxiety and depression, which are associated with worse HRQoL. There is an urgent need for psychological care in PCD, especially in adults with PCD and caregivers. https://bit.ly/3rmbgc9

Cite this article as: Verkleij M, Appelman I, Altenburg J, et al. Anxiety and depression in Dutch patients with primary ciliary dyskinesia and their caregivers: associations with health-related quality of life. ERJ Open Res 2021; 7: 00274-2021 [DOI: 10.1183/23120541.00274-2021].

\section{Abstract}

Background Primary ciliary dyskinesia (PCD) might be a risk factor for the development of anxiety and depression. This study investigated the associations between anxiety, depression and health-related quality of life (HRQoL) in individuals with PCD and their caregivers.

Methods Children, adolescents and adults with PCD and their caregivers were invited to participate in a mental health screening programme. During regular yearly outpatient visits, measures of anxiety (GAD-7), depression (PHQ-9), HRQoL (QOL-PCD), lung function (forced expiratory volume in $1 \mathrm{~s}\left(\mathrm{FEV}_{1}\right)$ ) and body mass index (BMI)) were collected and associations of anxiety, depression and HRQoL were estimated.

Results A total of 103 individuals participated in the mental health screening programme. Elevated levels of anxiety (scores $\geqslant 10$ on GAD-7) were found in $6 \%$ of adults ( $n=33), 14 \%$ of children $(n=7), 6 \%$ of adolescents ( $n=17)$ and $20 \%$ of caregivers ( $n=46,52 \%$ of mothers). Elevated depression levels (scores $\geqslant 10$ on PHQ-9) were found in $18 \%$ of adults, $14 \%$ of children, $6 \%$ of adolescents and $11 \%$ of caregivers. Anxiety and depression were associated with scales on the QOL-PCD. Mothers reported higher anxiety scores than fathers ( $30 \%$ versus $9 \%, \mathrm{p}=0.03$ ). A strong negative relationship was found between depression in caregivers and physical functioning (QOL-PCD) of the child. Anxiety and depression were not significantly associated with anxiety/depression in their child.

Conclusion This is the first study investigating anxiety and depression in individuals with PCD and their caregivers. Our results revealed elevated levels of anxiety and depression, which were associated with worse HRQoL. These results suggest the need for psychological support in PCD.

\section{Introduction}

Primary ciliary dyskinesia (PCD) is a chronic genetic disease with a worldwide prevalence of 1 in 10000 20000 people [1]. This is probably an underestimate, given that making the diagnosis is highly complex and many individuals are incorrectly diagnosed [2]. However, in some more or less genetically isolated and consanguineous populations like Volendam in the Netherlands, incidence can be as high as 1:400, with a carrier frequency of 1:10 [3]. PCD is associated with upper and lower respiratory tract infections due to structural and functional abnormalities of respiratory cilia [4]. Other clinical symptoms include situs inversus, neonatal respiratory distress and infertility $[4,5]$.

Individuals with a chronic respiratory disease are at increased risk for developing anxiety and depression symptoms [6-8]. These symptoms are associated with negative health outcomes, including poor treatment 
adherence, increased mortality, higher healthcare utilisation and costs, lower body mass index (BMI), decreased lung function (forced expiratory volume in $1 \mathrm{~s}\left(\mathrm{FEV}_{1}\right)$ ) and impaired health-related quality of life (HRQoL) [9-14]. In cystic fibrosis (CF), for example, a large-scale epidemiological study in nine countries indicated that rates of elevated anxiety and depression were two to three times higher than those found in community samples [14]. This led to the development of international mental health guidelines for CF, co-sponsored by the Cystic Fibrosis Foundation and the European Cystic Fibrosis Society [15, 16], which recommended annual mental health screening and treatment for people with $\mathrm{CF}$ and their caregivers. Moreover, caregivers of patients with chronic respiratory diseases also report higher rates of anxiety and depression than caregivers of healthy controls $[14,17,18]$.

Until now, research and clinical care in PCD has mainly focused on the genetics, the complexities of the diagnostic process and physical manifestations of the disease, with limited attention to psychosocial problems [19, 20]. The few studies that have examined the psychosocial impact of PCD have shown that these individuals report feelings of embarrassment, isolation and anxiety about the future [21, 22]. Children with PCD exhibited more behavioural problems than healthy controls, specifically internalising problems [23], and mothers of children with PCD reported higher levels of parenting stress and general stress compared to mothers of healthy children [23]. Moreover, HRQoL in individuals with PCD was worse than healthy controls [24]. Studies have also shown that both physical functioning and respiratory symptoms in adults with PCD decrease with age [21]. Recently, a disease-specific instrument for measuring HRQoL in PCD patients was developed and validated, the QOL-PCD [19, 21, 25, 26].

Given the limited literature on psychosocial adjustment in individuals with PCD and their caregivers, including the prevalence of psychological symptoms such as anxiety and depression, and their associations with HRQoL, a biopsychosocial approach was taken in this study. Thus, anxiety and depression may affect disease severity via mechanisms associated with poor treatment adherence and self-care (e.g. airway clearance, unhealthy diet, smoking) [10, 27]. Biological stress systems in the brain (e.g. sympathetic nervous system) are often dysregulated in individuals experiencing anxiety and depression, which might lead to increases in physical symptoms [28]. Evidence also supports this model in CF [29, 30]. Depression is associated with a chronic, low-grade inflammatory response and activation of cell-mediated immunity, as well as activation of the compensatory anti-inflammatory reflex system [31]. Studies in several chronic conditions have shown that depression, independent of disease, causes an increase in inflammation (e.g. coronary artery disease, diabetes). In patients with chronic respiratory conditions, inflammation is associated with pulmonary exacerbations and worsening disease [31-34]. Combined, these processes were expected to negatively impact HRQoL in people with PCD [35].

The psychological well-being of caregivers may also affect the psychological and physical health of children with PCD. Caregivers of children with chronic conditions report higher levels of anxiety and depression than caregivers of healthy children, which, in turn, has been associated with a higher risk of those children developing symptoms of anxiety and depression [14, 36, 37]. Psychological well-being of the caregiver has also been associated with disease severity. Parents experiencing depression, for example, may have more difficulty organising and assisting with treatments [29], decreasing adherence, which could potentially lead to more severe disease [29, 38]. On the other hand, more severe disease in the child has also been associated with higher rates of anxiety and depression in their caregivers [18].

To better inform the inclusion of psychological assessment and treatment into the multidisciplinary PCD care team, this study aimed to evaluate the complex associations between symptoms of anxiety and depression and HRQoL in individuals with PCD and their caregivers. We hypothesised that anxiety and depression in children and adults with PCD and their caregivers is more prevalent than reported for community samples, and that elevated symptoms of anxiety and/or depression will be associated with worse HRQoL. Moreover, we hypothesised that children with PCD who report elevated anxiety and/or depression scores and worse HRQoL will be more likely to have caregivers who also reported elevated psychological symptomatology.

\section{Methods}

\section{Study design}

This was a cross-sectional study conducted at a single PCD centre in the Netherlands. The Medical Ethics Committee of the VU University Medical Center (VUmc), Amsterdam, the Netherlands, approved the study.

\section{Participants}

From April 2019 to June 2020, all children (6-11 years), adolescents (12-17 years) and adults (aged $\geqslant 18$ years) with PCD, and caregivers of children with PCD (0-17 years) from Amsterdam University 
Medical Centers (Amsterdam UMC) were invited to participate in the study. PCD diagnosis was based on a combination of tests including high-speed videomicroscopy before and after cell culture, nasal nitric oxide (NO), transmission electron microscopy (TEM) and genetics. Similar to the iPCD cohort study [39], patients in this study can be divided into three subgroups based on diagnostic tests:

1. Definite PCD: patients with a clinical phenotype consistent with PCD and a hallmark TEM finding, and/or pathogenic biallelic PCD genetic mutations [40].

2. Probable PCD: clinical phenotype consistent with PCD and abnormal high-speed videomicroscopy findings and/or low nasal NO.

3. Clinical phenotypes consistent with PCD diagnosis in whom other possible diagnoses such as CF were excluded (none included in this study).

Adolescents and adults with PCD, as well as caregivers of children with PCD, provided written informed consent. Participants were excluded if they were unable to read and complete the questionnaires.

\section{Instruments}

Demographic and clinical variables

Demographic variables, such as age, gender, education level, marital status, ethnic background, comorbid diseases and previous and current psychological support were obtained from patients and caregivers during their annual clinic visit (see table 1) by a demographic questionnaire. Height and weight of the adults and children/adolescents with PCD were obtained from chart review to calculate their BMI. Spirometry values (lung function) were assessed using the Masterscreen (Jaeger ${ }^{\circledR}$, CareFusion Corporation). Before assessing lung function, short- or long-acting $\beta_{2}$-adrenergic agonists were stopped for $12 \mathrm{~h}$. FEV 1 was obtained for all adults with PCD and for children aged $\geqslant 6$ years. Normal values of $\mathrm{FEV}_{1}$ range between $80 \%$ and $120 \%$.

\section{Measures}

Generalised Anxiety Disorder 7-item (GAD-7, Dutch version [41])

The GAD-7 assesses anxiety symptoms. It consists of seven items, each scored as 0 ("not at all”), 1 ("several days"), 2 ("more than half of the days") or 3 ("nearly every day"). Total scores range from 0 to 21;

TABLE 1 Demographic characteristics of adults and children with PCD and parent caregivers ( $n=103)$

\begin{tabular}{|c|c|c|c|c|}
\hline & $\begin{array}{l}\text { Adults with PCD } \\
\qquad(n=33)\end{array}$ & $\begin{array}{c}\text { Children with } P C D \\
\text { (age } 6-17 \text { years; } n=24 \text { ) }\end{array}$ & $\begin{array}{l}\text { Mothers } \\
(n=24)\end{array}$ & $\begin{array}{c}\text { Fathers } \\
(n=22)\end{array}$ \\
\hline Female total group, $\mathrm{n}(\%)$ & $20(61)$ & $14(58)$ & & \\
\hline Age, years, mean \pm sD (range) & $38.3 \pm 18.0(18-77)$ & $13.3 \pm 3.3(7-17)$ & $42.8 \pm 6.1(32-55)$ & $44.7 \pm 5.2(34-54)$ \\
\hline Lung function $\left(\mathrm{FEV}_{1} \%\right.$ pred), mean $\pm \mathrm{SD}$ & $72.8 \pm 21.2$ & $78.7 \pm 13.4$ & & \\
\hline BMI, mean \pm SD & $24.2 \pm 4.2$ & $20.1 \pm 4.4$ & & \\
\hline Received psychological support in past year, $\mathrm{n}(\%)$ & $5(15)$ & $7(29)$ & $4(17)$ & $5(23)$ \\
\hline Currently receiving psychological support, n (\%) & $2(6)$ & $4(17)$ & $4(17)$ & $1(5)$ \\
\hline Need for psychological support ${ }^{\#}, \mathrm{n}(\%)$ & $5(15)$ & $3(15)$ & $4(17)$ & $1(5)$ \\
\hline \multicolumn{5}{|l|}{ Relational status, $\mathrm{n}$} \\
\hline Alone & 9 & & 0 & 0 \\
\hline Married/living together & 19 & & 23 & 20 \\
\hline Divorced & 0 & & 1 & 2 \\
\hline Widowed & 1 & & 0 & 0 \\
\hline Other & 1 & & & \\
\hline \multicolumn{5}{|l|}{ Ethnic background, $n$} \\
\hline Dutch & 27 & & 22 & 21 \\
\hline Turkish & 1 & & 1 & 1 \\
\hline Other & 1 & & 1 & 0 \\
\hline \multicolumn{5}{|l|}{ Education level", $n$} \\
\hline Low & 10 & & 3 & 1 \\
\hline Middle & 3 & & 7 & 12 \\
\hline High & 14 & & 13 & 9 \\
\hline Currently working, $\mathrm{n}$ & 22 & & 22 & 22 \\
\hline Other chronic illness, $\mathrm{n}$ & 9 & & 6 & 5 \\
\hline
\end{tabular}


scores of 5, 10 and 15 points are used as cut-off points from a general population for mild, moderate and severe anxiety, respectively. The GAD-7 has excellent psychometric properties, including strong internal consistency (Cronbach's $\alpha=0.85$ ) and convergent validity with the Diagnostic and Statistical Manual of Mental Disorder, 5th Edition (DSM-5) diagnostic criteria. Using a cut-off score of 10 or higher, sensitivity was 0.89 and specificity 0.82 [41, 42]. The minimal important difference score of 4 points has been established for the GAD-7 [43]. In this study, the moderate score of the GAD-7 $(\geqslant 10)$ was used as the clinical cut-off.

\section{Patient Health Questionnaire-9 (PHQ-9, Dutch version [44])}

The PHQ-9 assesses depressive symptoms and consists of nine items corresponding to DSM-5 diagnostic criteria for major depression. It is one of the most widely used depression screeners, is free and available in all major world languages. It has demonstrated strong psychometric properties. Items are scored on a four-point Likert scale: 0 (“not at all”), 1 (“several days”), 2 (“more than half of the days”) or 3 ("nearly every day”). Scores range from 0 to 27 , with higher scores indicating more severe depressive symptoms. Scores can be divided according to level of severity: "minimal" (scores 0-4), "mild" (scores 5-9), "moderate" (scores 10-14), "moderately severe" (scores 15-19) and "severe" (scores 20-27), with cut-off scores determined from a general population. The PHQ-9 also assesses suicidal ideation (question \#9) which can accompany more severe depressive symptoms. Studies have shown that using a threshold score of 10 or higher has a sensitivity of 0.85 and specificity of 0.89 [45]. The minimal clinically important difference score of 5 points has been established [46]. The moderate score of the PHQ-9 $(\geqslant 10)$ was used as the clinical cut-off.

Scores on the GAD-7 and PHQ-9 were categorised twice. First, scores were categorised on the basis of severity as follows: 0 (scores $\leqslant 4$; "minimal”), 1 (scores 5-9; “mild”), 2 (scores 10-14; "moderate”) or 3 (scores $\geqslant 15$; "severe"). Then, all scores of $\geqslant 10$ on the GAD-7 and PHQ-9 were considered clinically elevated (in accordance with the International Committee on Mental Health (ICMH) recommendations) and manually scored as "1" (“elevated”). All scores $<10$ were manually scored as 0 ("not elevated”).

In children aged 6-11 years, the GAD-7 and PHQ-9 were filled in by the parent caregiver assessing their child's behaviour. Adolescents (12-17 years) filled in the GAD-7 and PHQ-9 by themselves.

\section{Quality of Life for Primary Ciliary Dyskinesia (QOL-PCD; Dutch version [25, 26])}

The QOL-PCD is a profile measure of HRQoL for people with PCD, assessing quality of life across several domains. Different versions of the QOL-PCD have been developed for children (6-11 years; 34 items, 7 scales), adolescents (12-17 years; 35 items, 9 scales) and adults (aged $\geqslant 18$ years; 40 items, 10 scales). The seven scales that were included in all three versions of the QOL-PCD were: Physical Functioning, Emotional Functional, Social Functioning, Treatment Burden, Upper Respiratory Symptoms, Lower Respiratory Symptoms, and Ear and Hearing Symptoms. Other scales for adolescents and adults included: Role Functioning and Vitality (adolescents and adult version) and Health Perceptions (adult version). The different versions of the QOL-PCD have all demonstrated good reliability and validity [19, 21].

\section{Procedure}

Following the CF mental health guidelines [15], the procedure was as follows:

1. Adults, children and adolescents with PCD and caregivers of children with PCD received the questionnaires (GAD-7, PHQ-9 and QOL-PCD) from the PCD nurse at their annual medical check-up. The questionnaires were either completed immediately and returned to the PCD nurse, or filled in at home and returned by mail.

2. Upon receipt of the questionnaires, the psychologist immediately checked the PHQ-9 for suicidal ideation. If this item was endorsed, the psychologist determined the severity by using the Columbia Suicide Severity Rating Scale (C-SSRS). If indicated, a referral to the psychiatrist in attendance at the hospital was made.

3. The other measures were scored within a week.

4. Psychologists called the participants to discuss the results of the GAD-7, PHQ-9 and QOL-PCD, and a referral plan to a healthcare provider was developed if needed.

\section{Statistical analysis}

All statistical analyses were performed with IBM SPSS Statistics for Windows, Version 26. p-values of $<0.05$ (two-sided) were considered statistically significant. For missing values on the QOL-PCD, the median value of the corresponding scale was entered.

Descriptive statistics were used to evaluate and summarise the demographic and clinical characteristics of the participants as well as the mean anxiety, depression and HRQoL scores. One-way ANOVA was used to 
examine differences between the three groups of adults, adolescents and children with PCD and independent t-tests were used to examine differences in caregivers' scores of anxiety, depression and HRQoL.

Regression analyses were used to analyse the univariable associations between anxiety, depression and HRQoL across the three groups. Mixed model analyses were used to estimate the associations between anxiety, depression and HRQoL in children with anxiety and depression and their caregivers. Mixed model analysis was used to take into account the dependency of the observations for two caregivers with one child. All regression analyses were adjusted for age, gender, BMI and lung function.

\section{Results}

\section{Participant characteristics}

In total, 43 adults and 45 children/adolescents with a confirmed diagnosis of PCD and at least one primary caregiver were invited to participate in the study. Total inclusion rate of participants with PCD was $66 \%$. Ten adults and 19 children/adolescents with PCD did not respond or did not return the questionnaires; two children were unable to read in Dutch (2\%). This resulted in a total of 103 participants: 33 (of 43) adults with PCD (77\%); 24 (of 43) children/adolescents with PCD (7 children 6-11 years. and 17 adolescents 12-17 years) (56\%); and 46 caregivers (24 mothers and 22 fathers of children/adolescents with PCD). Of the 24 children/adolescents with PCD, all mothers and fathers participated (two adolescents had no father). Based on diagnostic tests performed, 26 out of 33 adults (79\%) and 24 out of 27 children/adolescents (89\%) were classified as definite PCD. The remaining patients were classified as probable PCD.

Table 1 presents the demographic characteristics of the adults and children with PCD and their caregivers. Mean lung function of adults and children with PCD (6-11 years) was below average $(<80 \%)$; mean BMI of adults with PCD was considered healthy (between 18.5 and $24.9 \mathrm{~kg} \cdot \mathrm{m}^{-2}$ ).

In the demographic questionnaire, 21\% of adults with PCD filled in that they are currently receiving or have received psychological support in the past year, and $15 \%$ need psychological support. In children with PCD (6-17 years), 46\% are currently receiving or have received psychological support in the past year, and 15\% need psychological support. Thirty-four per cent of mothers and 28\% of fathers are currently receiving or have received psychological support in the past year; $17 \%$ of mothers emphasised the need for psychological support and 5\% of fathers need psychological support.

\section{Prevalence of anxiety and depression}

We hypothesised that anxiety and depression in individuals with PCD and their caregivers would be more prevalent than among community samples. Table 2 shows the classification of anxiety and depression scores in adults and children/adolescents with PCD and caregivers. Clinically elevated symptoms of anxiety were reported by $6 \%$ of adults, $14 \%$ of children and $6 \%$ of adolescents with PCD and $30 \%$ of mothers and $9 \%$ of fathers. Clinically elevated symptoms of depression were reported by $18 \%$ of adults, $14 \%$ of children and $6 \%$ of adolescents with PCD, $12 \%$ of mothers and $9 \%$ of fathers.

TABLE 2 Classification of anxiety and depression scores in adults, children and adolescents with PCD and caregivers ( $n=103)$

Adults with PCD

$(n=33)$
Children with PCD

(age 6-11 years, $n=7$ )
Adolescents with PCD

(age $12-17$ years, $n=17$ )
Mothers $(n=24)$
Fathers $(n=22)$

\begin{tabular}{|c|c|c|c|c|c|}
\hline \multicolumn{6}{|l|}{ Anxiety (GAD-7), n (\%) } \\
\hline Normal range (score $\leqslant 4$ ) & $18(55)$ & $6(86)$ & $5(29)$ & $12(50)$ & $17(77)$ \\
\hline Mild range (score 5-9) & $13(39)$ & 0 & $11(65)$ & $5(21)$ & $3(14)$ \\
\hline Moderate range (score $10-14$ ) & $2(6)$ & $1(14)$ & $1(6)$ & $3(13)$ & $2(9)$ \\
\hline Severe range $($ score $\geqslant 15)$ & 0 & 0 & 0 & $4(17)$ & 0 \\
\hline \multicolumn{6}{|l|}{ Depression (PHQ-9), n (\%) } \\
\hline Normal range (score $\leqslant 4)$ & $16(49)$ & $4(57)$ & $7(41)$ & $12(50)$ & $15(68)$ \\
\hline Mild range (score 5-9) & $11(33)$ & $2(29)$ & $9(53)$ & $9(38)$ & $5(23)$ \\
\hline Moderate range (score $10-14$ ) & $5(15)$ & $1(14)$ & 0 & $1(4)$ & $2(9)$ \\
\hline Severe range $($ score $\geqslant 15$ ) & $1(3)$ & 0 & $1(6)$ & $2(8)$ & 0 \\
\hline
\end{tabular}




\begin{tabular}{|c|c|c|c|c|c|c|c|c|c|c|}
\hline & \multicolumn{2}{|c|}{$\begin{array}{l}\text { Adults with } \\
\text { PCD }(n=33)\end{array}$} & \multicolumn{2}{|c|}{$\begin{array}{c}\text { Adolescents } \\
\text { with PCD (age } \\
\begin{array}{c}\text { 12-17 years, } \\
n=17)\end{array}\end{array}$} & \multicolumn{2}{|c|}{$\begin{array}{l}\text { Children with } \\
\text { PCD (age } \\
\text { 6-11 years, } \\
n=7 \text { ) }\end{array}$} & \multicolumn{2}{|c|}{$\begin{array}{l}\text { Mothers } \\
(n=24)\end{array}$} & \multicolumn{2}{|c|}{$\begin{array}{l}\text { Fathers } \\
(n=22)\end{array}$} \\
\hline & Mean & (SD) & Mean & (SD) & Mean & (SD) & Mean & (SD) & Mean & (SD) \\
\hline Anxiety $(G A D-7)^{\#}$ & 4.3 & $(3.1)$ & 5.4 & $(2.9)$ & 3.0 & $(4.4)$ & 6.2 & $(6.2)$ & 3.0 & (3.3) \\
\hline Depression (PHQ-9) & 5.6 & $(4.2)$ & 5.5 & (3.5) & 3.6 & $(4.2)$ & 5.4 & (4.7) & 3.1 & (3.8) \\
\hline \multicolumn{11}{|c|}{ Health-related quality of life (QOL-PCD) domains } \\
\hline Physical Functioning & 65.3 & $(29.2)$ & 87.5 & $(15.0)$ & 84.8 & $(14.8)$ & & & & \\
\hline Emotional Functioning & 81.9 & $(13.1)$ & 73.4 & $(7.6)$ & 76.2 & $(13.1)$ & & & & \\
\hline Social Functioning & 34.7 & $(27.0)$ & 62.5 & $(19.7)$ & 91.4 & $(10.7)$ & & & & \\
\hline Treatment Burden & 60.9 & $(23.2)$ & 74.8 & $(22.4)$ & 80.0 & $(26.1)$ & & & & \\
\hline Role Functioning & 66.2 & $(25.4)$ & 86.8 & $(15.3)$ & & & & & & \\
\hline Vitality & 55.9 & (19.3) & 60.4 & $(14.6)$ & & & & & & \\
\hline Health Perceptions & 50.8 & $(22.6)$ & & & & & & & & \\
\hline Upper Respiratory Symptoms & 55.1 & $(21.6)$ & 69.6 & $(19.3)$ & 75.2 & $(17.5)$ & & & & \\
\hline Lower Respiratory Symptoms & 51.3 & $(18.7)$ & 58.9 & $(19.1)$ & 73.0 & $(10.4)$ & & & & \\
\hline Hearing Symptoms & 68.2 & $(32.9)$ & 83.9 & $(18.1)$ & 86.9 & $(10.6)$ & & & & \\
\hline
\end{tabular}

Additional analyses computed for adolescents with PCD and their caregivers showed that 6\% of adolescents scored in the elevated range (on either GAD-7 or PHQ-9), as did $29 \%$ of mothers and $14 \%$ of fathers.

Anxiety, depression and HRQoL

Table 3 shows mean anxiety, depression and HRQoL scores of adults, adolescents and children with PCD and caregivers. No significant differences were found in mean anxiety and depression scores between adults, adolescents and children with PCD. Mothers reported more symptoms of anxiety than fathers $(\mathrm{p}=0.03)$.

The HRQoL domains Physical Functioning, Social Functioning, Treatment Burden, Role Functioning and Symptoms were worse with increasing age.

TABLE 4 Unstandardised regression coefficients, confidence interval and p-values regarding the associations between anxiety, depression and health-related quality of life in adults with PCD ( $n=33)$

Anxiety (GAD-7)

\begin{tabular}{lcc}
\hline Health-related quality of life (QOL-PCD) & domains \\
Physical Functioning & $-2.03(-6.83$ to 2.77$) p=0.39$ & -1.74 (-5.18 to 1.71$) p=0.31$ \\
\hline Emotional Functioning & $-2.95(-5.21$ to -0.70$) p=0.01$ & $-0.84(-1.70$ to 1.54$) p=0.92$ \\
Social Functioning & $3.93(-2.56$ to 10.43$) p=0.22$ & $-3.93(-8.60$ to 0.73$) p=0.10$ \\
Treatment Burden & $4.37(-2.89$ to 11.62$) p=0.22$ & $-4.12(-8.97$ to 0.73$) p=0.09$ \\
Role Functioning & $-0.96(-5.14$ to 3.24$) p=0.64$ & -3.55 (-6.55 to -0.54$) p=0.02$ \\
Vitality & $-1.18(-4.42$ to 2.05$) p=0.46$ & $-2.35(-4.67$ to -0.02$) p=0.05$ \\
Health Perceptions & $-3.11(-6.93$ to 0.71$) p=0.11$ & $-1.53(-4.10$ to 1.04$) p=0.23$ \\
Upper Respiratory Symptoms & $0.59(-3.74$ to 4.91$) p=0.78$ & $-2.67(-5.78$ to 0.43$) p=0.09$ \\
Lower Respiratory Symptoms & $-1.01(-4.95$ to 2.92$) p=0.60$ & $-0.75(-3.57$ to 2.08$) p=0.59$ \\
Hearing Symptoms & $2.05(-4.76$ to 8.86$) p=0.54$ & $-3.29(-8.17$ to 1.61$) p=0.18$ \\
\hline PCD: primary ciliary dyskinesia; GAD-7: Generalised Anxiety Disorder 7-Item; PHQ-9: Patient Health \\
Questionnaire-9; QOL-PCD: Quality of Life Instrument for Primary Ciliary Dyskinesia.
\end{tabular}


TABLE 5 Unstandardised regression coefficients, confidence interval and p-values regarding the associations between anxiety, depression and health-related quality of life in adolescents and children with PCD

\begin{tabular}{|c|c|c|c|c|}
\hline & \multicolumn{2}{|c|}{ Adolescents with PCD (12-17 years), $n=17$} & \multicolumn{2}{|c|}{ Children with PCD (6-11 years), $n=7$} \\
\hline & Anxiety (GAD-7) & Depression (PHQ-9) & Anxiety (GAD-7) & Depression (PHQ-9) \\
\hline \multicolumn{5}{|c|}{ Health-related quality of life (QOL-PCD) domains } \\
\hline Physical Functioning & $\begin{array}{c}2.55(-1.47 \text { to } 6.57) \\
p=0.19\end{array}$ & $\begin{array}{c}-4.79(-8.16 \text { to }-1.42) \\
\mathrm{p}=0.01\end{array}$ & $\begin{array}{c}-2.36(-9.80 \text { to } 5.08) \\
p=0.31\end{array}$ & $\begin{array}{c}0.11(-8.92 \text { to } 9.15) \\
p=0.96\end{array}$ \\
\hline Emotional Functioning & $\begin{array}{c}-0.93(-4.63 \text { to } 4.45) \\
p=0.96\end{array}$ & $\begin{array}{c}-0.23(-4.04 \text { to } 3.58) \\
p=0.90\end{array}$ & $\begin{array}{c}-2.91(-6.69 \text { to } 0.87) \\
p=0.08\end{array}$ & $\begin{array}{c}0.71(-3.88 \text { to } 5.30) \\
p=0.57\end{array}$ \\
\hline Social Functioning & $\begin{array}{c}-2.95(-14.62 \text { to } 8.73) \\
p=0.58\end{array}$ & $\begin{array}{c}2.59(-7.21 \text { to } 12.38) \\
p=0.57\end{array}$ & $\begin{array}{c}0.30(-7.98 \text { to } 8.58) \\
p=0.89\end{array}$ & $\begin{array}{c}0.76(-9.30 \text { to } 10.82) \\
p=0.78\end{array}$ \\
\hline Treatment Burden & $\begin{array}{c}2.45(-21.66 \text { to } 26.55) \\
p=0.82\end{array}$ & $\begin{array}{c}-3.27(-23.07 \text { to } 16.53) \\
p=0.71\end{array}$ & $\begin{array}{c}-0.01(-16.16 \text { to } 16.13) \\
p=0.99\end{array}$ & $\begin{array}{c}-3.58(-23.18 \text { to } 16.03) \\
p=0.52\end{array}$ \\
\hline Role Functioning & $\begin{array}{c}2.75(-4.73 \text { to } 10.22) \\
p=0.43\end{array}$ & $\begin{array}{c}-3.78(-10.05 \text { to } 2.49) \\
p=0.21\end{array}$ & & \\
\hline Vitality & $\begin{array}{c}3.21(-2.29 \text { to } 8.71) \\
p=0.22\end{array}$ & $\begin{array}{c}-5.82(-10.43 \text { to }-1.21) \\
p=0.02\end{array}$ & & \\
\hline Upper Respiratory Symptoms & $\begin{array}{c}3.28(-6.04 \text { to } 12.61) \\
p=0.45\end{array}$ & $\begin{array}{c}-2.23(-10.05 \text { to } 5.59) \\
p=0.54\end{array}$ & $\begin{array}{c}-2.30(-15.92 \text { to } 11.32) \\
\mathrm{p}=0.54\end{array}$ & $\begin{array}{c}-0.30(-16.85 \text { to } 16.24) \\
p=0.95\end{array}$ \\
\hline Lower Respiratory Symptoms & $\begin{array}{c}0.40(-6.63 \text { to } 7.42) \\
p=0.90\end{array}$ & $\begin{array}{c}0.42(-5.48 \text { to } 6.32) \\
p=0.88\end{array}$ & $\begin{array}{c}1.78(-3.67 \text { to } 7.22) \\
p=0.30\end{array}$ & $\begin{array}{c}-3.40(-10.02 \text { to } 3.22) \\
p=0.16\end{array}$ \\
\hline Hearing Symptoms & $\begin{array}{c}-2.13(-11.88 \text { to } 7.62) \\
p=0.63\end{array}$ & $\begin{array}{c}1.80(-6.38 \text { to } 9.98) \\
p=0.63\end{array}$ & $\begin{array}{c}-1.43(-10.51 \text { to } 7.64) \\
p=0.57\end{array}$ & $\begin{array}{c}0.54(-10.48 \text { to } 11.56) \\
p=0.85\end{array}$ \\
\hline
\end{tabular}

PCD: primary ciliary dyskinesia; GAD-7: Generalised Anxiety Disorder 7-Item; PHQ-9: Patient Health Questionnaire-9; QOL-PCD: Quality of Life Instrument for Primary Ciliary Dyskinesia.

As hypothesised, elevated symptoms of anxiety and/or depression were associated with worse HRQoL. In adults, anxiety was significantly associated with a worse score on the QOL-PCD domain Emotional Functioning. Depression was significantly associated with worse scores on the QOL-PCD domains Role Functioning and Vitality (table 4). In adolescents, higher depression scores were significantly associated with worse scores on the QOL-PCD domains Physical Functioning and Vitality. In children (6-11 years), anxiety and depression were not significantly associated with their scores on any of the QOL-PCD domains (table 5).

\section{Associations between caregivers and children}

Thirdly, we hypothesised that children/adolescents with PCD who report elevated anxiety and/or depression scores and worse HRQoL will be more likely to have caregivers who also reported elevated symptoms of anxiety and/or depression. Table 6 shows the results of the mixed model analyses regarding the association between anxiety and depression in caregivers and HRQoL in their children with PCD. A strong negative relationship was found between depression in caregivers and the QOL-PCD domain Physical Functioning in their child (table 6). Parents who reported higher depressive symptoms had children/adolescents who reported worse Physical Functioning. All other relationships between anxiety and depression in caregivers of children/adolescents with PCD and HRQoL (domains of the QOL-PCD) of their children/adolescents were not statistically significant.

The results of the mixed model analyses regarding the association between anxiety and depression in children with PCD and anxiety and depression in their caregivers do not indicate significant associations (table 7), although there was a positive relationship for depression, which trended toward significance ( $p=0.052$ for the crude relationship). When adjusted for age, gender, BMI and lung function, this association did not reach statistical significance.

\section{Discussion}

To our knowledge, this is the first study to examine the prevalence of anxiety and depression, and its associations with HRQoL, in people with PCD and parent caregivers. Results showed that elevated symptoms of anxiety and depression were prevalent in people with PCD and their caregivers. In particular, nearly half of the adults with PCD and half of the mothers of children/adolescents with PCD reported psychological symptoms in the mild to severe range. Anxiety and depression scores in children/adolescents 


\begin{tabular}{|c|c|c|c|}
\hline Health-related quality of life (QOL-PCD) domains & & Anxiety (GAD-7) & Depression (PHQ-9) \\
\hline \multirow[t]{2}{*}{ Physical Functioning } & Crude & $-0.12(-0.46$ to 0.22$) p=0.48$ & $-0.39(-0.69$ to -0.09$) p=0.01$ \\
\hline & Adjusted & $-0.21(-0.67$ to 0.26$) p=0.38$ & $-0.50(-0.82$ to -0.18$) p=0.002$ \\
\hline \multirow[t]{2}{*}{ Emotional Functioning } & Crude & $0.27(-0.11$ to 0.66$) p=0.17$ & $-0.13(-0.49$ to 0.23$) p=0.47$ \\
\hline & Adjusted & $0.32(-0.10$ to 0.74$) p=0.14$ & $-0.15(-0.51$ to 0.20$) p=0.40$ \\
\hline \multirow[t]{2}{*}{ Treatment Burden } & Crude & $0.24(-0.13$ to 0.61$) p=0.21$ & $-0.17(-0.49$ to 0.16$) p=0.32$ \\
\hline & Adjusted & $0.25(-0.13$ to 0.63$) p=0.20$ & $-0.18(-0.50$ to 0.14$) p=0.27$ \\
\hline \multirow[t]{2}{*}{ Social Functioning } & Crude & $0.05(-0.31$ to 0.41$) p=0.80$ & $0.10(-0.21$ to 0.41$) p=0.53$ \\
\hline & Adjusted & $0.07(-0.45$ to 0.59$) p=0.79$ & $0.09(-0.38$ to 0.56$) p=0.72$ \\
\hline \multirow[t]{2}{*}{ Upper Respiratory Symptoms } & Crude & $0.10(-0.27$ to 0.46$) p=0.61$ & $0.02(-0.31$ to 0.35$) p=0.91$ \\
\hline & Adjusted & $0.21(-0.37$ to 0.78$) p=0.49$ & $0.15(-0.29$ to 0.60$) p=0.49$ \\
\hline \multirow[t]{2}{*}{ Lower Respiratory Symptoms } & Crude & $0.07(-0.30$ to 0.44$) p=0.71$ & $0.08(-0.25$ to 0.42$) p=0.63$ \\
\hline & Adjusted & $0.28(-0.56$ to 1.11$) p=0.52$ & $0.27(-0.27$ to 0.81$) p=0.33$ \\
\hline \multirow[t]{2}{*}{ Hearing Symptoms } & Crude & $-0.12(-0.44$ to 0.19$) p=0.45$ & $-0.03(-0.38$ to 0.32$) p=0.85$ \\
\hline & Adjusted & $-0.13(-0.44$ to 0.19$) p=0.44$ & $-0.08(-0.43$ to 0.28$) p=0.68$ \\
\hline
\end{tabular}

Adjusted: adjusted for gender, age, FEV 1 and BMI. PCD: primary ciliary dyskinesia; GAD-7: Generalised Anxiety Disorder 7-Item; PHQ-9: Patient Health Questionnaire-9; FEV 1 : forced expiratory volume in $1 \mathrm{~s}$, expressed as percentage of the predicted value; BMI: body mass index; QOL-PCD: Quality of Life Instrument for Primary Ciliary Dyskinesia.

and adults with PCD were associated with worse HRQoL, and depression in caregivers was associated with worse Physical Functioning (HRQoL) in their child/adolescent.

In accordance with our hypothesis, elevated symptoms of anxiety and depression were prevalent in children/adolescents and adults with PCD and caregivers. However, these scores differ from the CF population in a previous study of a sample of Dutch CF patients who are cared for at the same academic medical hospital (VUmc) [16]. Our study showed that 6\% of adolescents with PCD reported elevated scores in comparison to $12 \%$ of adolescents with CF. The lower rates in PCD might be associated with the high burden of disease severity in adolescents with CF. Even though both conditions are chronic and complex, treatment of CF is more complex, time-consuming and burdensome than treatment of PCD [47, 48]. In addition, life expectancy is more severely reduced in CF. In contrast, social functioning appears to be negatively affected in both PCD and CF, possibly because individuals with both diseases report feeling stigmatised and/or socially isolated [22, 49].

Importantly, symptoms of anxiety and depression in parents of adolescents with PCD were higher than those reported for parents of adolescents with CF, using the same screening measures [16]. Nearly 30\% of mothers caring for adolescents with PCD reported elevated depression/anxiety compared to $13 \%$ of mothers of adolescents with CF and 14\% of fathers caring for an adolescent with PCD compared to $9 \%$ of fathers of adolescents with CF.

The high rates of anxiety and depression documented in this study are in line with previous studies, suggesting that caregivers of chronically ill children experience more anxiety and depression symptoms

TABLE 7 Standardised regression coefficients and 95\% confidence intervals (in brackets) regarding the association between anxiety and depression in children ( $\mathrm{n}=24$; independent variables) with PCD and anxiety and depression in their caregivers ( $n=46$; outcome variables)

\begin{tabular}{|c|c|c|c|}
\hline & & Anxiety (GAD-7) & Depression (PHQ-9) \\
\hline \multirow[t]{2}{*}{ Anxiety (GAD-7) } & Crude & $0.04(-0.26$ to 0.34$) p=0.79$ & \\
\hline & Adjusted & $0.14(-0.22$ to 0.49$) \mathrm{p}=0.45$ & \\
\hline \multirow[t]{2}{*}{ Depression (PHQ-9) } & Crude & & $0.28(-0.03$ to 0.58$) \mathrm{p}=0.052$ \\
\hline & Adjusted & & $0.24(-0.09$ to 0.57$) p=0.16$ \\
\hline
\end{tabular}


than parents of healthy children, especially mothers $[37,49]$. In accordance with these studies, mothers of children/adolescents with PCD reported more symptoms of anxiety than fathers; $30 \%$ of mothers reported anxiety scores in the moderate to severe range, indicating that these mothers are at high risk. This may be due to the lack of psychological support embedded into the multidisciplinary PCD team, the lack of well-organised patient organisations and foundations, and support among patients and families.

Anxiety and/or depression symptoms in children/adolescents and adults with PCD were negatively associated with worse HRQoL, thereby confirming our hypothesis. The association of anxiety and depression with worse HRQoL is supported by previous studies examining other chronic respiratory diseases [10, 12]. Our results indicate that this is true of not just Physical Functioning and Vitality in those with PCD, but also affects Emotional and Role Functioning. Given that anxiety and depression were negatively related to HRQoL, measuring and treating these symptoms might serve as a preventive treatment. Future research should investigate whether identifying and treating psychological symptoms might reduce the negative impact on HRQoL.

Studies of CF and asthma [14, 38] have shown that anxiety and/or depression in caregivers were associated with similar elevation in their children/adolescents. In this study, results showed that depression in caregivers was associated with Physical Functioning of the child on the QOL-PCD. Due to our study design, causality cannot be established. However, there might be a bidirectional relationship [50], indicating a "family systems" approach, involving both children/adolescents with PCD and their caregivers in clinical care. The importance of this approach is highlighted by previous studies indicating that worse emotional well-being in caregivers is negatively related to treatment adherence and disease severity in their children [38, 51]. More adults reported elevated symptoms of depression (18\%) than anxiety (6\%). Depression is seen as far more harmful for disease management and health outcomes. In CF, depression has been associated with earlier mortality, more frequent exacerbations and lower lung function [30]. Therefore, it is important to screen and treat depression at an early stage.

Strengths and limitations should be considered. Screening measures such as the GAD-7 and PHQ-9 are not a full diagnostic clinical interview, and these screening measures are not sufficient for a diagnosis. However, both the GAD-7 and PHQ-9 have proven to be effective and valid in screening anxiety and depression symptoms and are the recommended screening instruments according to the ICMH guidelines and most major international guidelines (e.g. National Institute for Health and Care Excellence guidelines, USPTF) $[15,42,45]$. A limitation of the study is that parents completed the GAD-7 and PHQ-9 for children under the age of 12, because there are very few well-established screeners for this age group.

Importantly, the results of our study may represent a first step towards more multidisciplinary clinical care of PCD. The use of a disease-specific instrument (QOL-PCD) provided a great deal of insight into the impact of PCD on daily functioning. Given the rare nature of this disease, a relatively large number of people with PCD and parent caregivers were included in this study. However, a larger sample size would increase statistical power of our analyses and improve their generalisability. Due to the cross-sectional design of the study, without multiple measurements over time, no assumptions can be made about the causal directions of these relationships. In the future, a longitudinal repeated-measures design should investigate whether anxiety and depression are associated with other important health variables and outcomes, such as treatment adherence, mortality, healthcare utilisation and costs [10, 13].

\section{Clinical implications}

Our results show that there is an urgent need for psychological care in the PCD populations, especially in adults with PCD and parents of children/adolescents with PCD. Psychological care is already embedded in CF care at many CF centres in the Netherlands, in which a psychologist/social worker is part of the multidisciplinary team. This model might work well in PCD too. In the CF mental health guidelines, annual mental health screening and treatment is recommended beginning at age 12 for adolescents with $\mathrm{CF}$ and for parents of children 0 through 17 years [15]. National implementation of these guidelines has been highly successful [52], partly because of the development of a mental health "toolbox" and additional mental health training sessions at national conferences. The CF ICMH guidelines can be implemented in clinical PCD care as well, if the appropriate mental health experts are integrated into the care team. Evaluating the possibilities of implementing these screening recommendations in clinical practice should be a focus of future research. Moreover, additional studies are needed to identify and address factors that might influence mental health, disease severity and HRQoL in individuals with PCD and their caregivers.

Provenance: Submitted article, peer reviewed. 
Acknowledgements: We thank the children, adolescents and adults with PCD and their caregivers for their cooperation, and the personnel for their help with recruitment.

Conflict of interest: None declared.

\section{References}

1 Mirra V, Werner C, Santamaria F. Primary ciliary dyskinesia: an update on clinical aspects, genetics, diagnosis, and future treatment strategies. Front Pediatr 2017; 5: 135

2 Behan L, Galvin AD, Rubbo B, et al. Diagnosing primary ciliary dyskinesia: an international patient perspective. Eur Respir J 2016; 48: 1096-1107.

3 Onoufriadis A, Paff T, Antony $\mathrm{D}$, et al. Splice-site mutations in the axonemal outer dynein arm docking complex gene CCDC114 cause primary ciliary dyskinesia. Am J Hum Genet 2013; 92: 88-98.

4 Goutaki M, Meier AB, Halbeisen FS, et al. Clinical manifestations in primary ciliary dyskinesia: systematic review and meta-analysis. Eur Respir J 2016; 48: 1081-1095.

5 Behan L, Dimitrov BD, Kuehni CE, et al. PICADAR: a diagnostic predictive tool for primary ciliary dyskinesia. Eur Respir J 2016; 47: 1103-1112.

6 Ryu YJ, Chun EM, Lee JH, et al. Prevalence of depression and anxiety in outpatients with chronic airway lung disease. Korean J Intern Med 2010; 25: 51-57.

7 Stoop $\mathrm{CH}$, Nefs G, Pommer AM, et al. Effectiveness of a stepped care intervention for anxiety and depression in people with diabetes, asthma or COPD in primary care: a randomized controlled trial. J Affect Disord 2015; 184: $269-276$.

8 Verkleij M, Beelen A, van Ewijk BE, et al. Multidisciplinary treatment in children with problematic severe asthma: a prospective evaluation. Pediatr Pulmonol 2017; 52: 588-597.

9 Janssen DJ, Spruit MA, Leue C, et al. Symptoms of anxiety and depression in COPD patients entering pulmonary rehabilitation. Chron Respir Dis 2010; 7: 147-157.

10 Knudsen KB, Pressler T, Mortensen LH, et al. Associations between adherence, depressive symptoms and health-related quality of life in young adults with cystic fibrosis. SpringerPlus 2016; 5: 1216.

11 Lu Y, Mak KK, Van Bever HP, et al. Prevalence of anxiety and depressive symptoms in adolescents with asthma: a meta-analysis and meta-regression. Pediatr Allergy Immunol 2012; 23: 707-715.

12 Riekert KA, Bartlett SJ, Boyle MP, et al. The association between depression, lung function, and health-related quality of life among adults with cystic fibrosis. Chest 2007; 132: 231-237.

13 Panagioti M, Scott C, Blakemore A, et al. Overview of the prevalence, impact, and management of depression and anxiety in chronic obstructive pulmonary disease. Int J Chron Obstruct Pulmon Dis 2014; 9: 1289-1306.

14 Quittner AL, Goldbeck L, Abbott J, et al. Prevalence of depression and anxiety in patients with cystic fibrosis and parent caregivers: results of The International Depression Epidemiological Study across nine countries. Thorax 2014; 69: 1090-1097.

15 Quittner AL, Abbott J, Georgiopoulos AM, et al. International Committee on Mental Health in Cystic Fibrosis: Cystic Fibrosis Foundation and European Cystic Fibrosis Society consensus statements for screening and treating depression and anxiety. Thorax 2016; 71: 26-34.

16 Verkleij M, de Winter D, Hurley MA, et al. Implementing the International Committee on Mental Health in Cystic Fibrosis (ICMH) guidelines: screening accuracy and referral-treatment pathways. J Cyst Fibros 2018; 17: 821-827.

17 Besier T, Goldbeck, L. Anxiety and depression in adolescents with CF and their caregivers. J Cyst Fibros 2011; 10: 435-442.

18 Easter G, Sharpe L, Hunt, CJ. Systematic review and meta-analysis of anxious and depressive symptoms in caregivers of children with asthma. J Pediatr Psychol 2015; 40: 623-632.

19 Behan L, Leigh MW, Dell SD, et al. Validation of pediatric health-related quality of life instruments for primary ciliary dyskinesia (QOL-PCD). Pediatr Pulmonol 2019; 54: 2011-2020.

20 Daniels MLA, Noone PG. Genetics, diagnosis and future treatment strategies for primary ciliary dyskinesia. Expert Opin Orphan Drugs 2015; 3: 31-44.

21 Behan L, Leigh MW, Dell SD, et al. Validation of a health-related quality of life instrument for primary ciliary dyskinesia (QOL-PCD). Thorax 2017; 72: 832-839.

22 Whalley S, McManus IC. Living with primary ciliary dyskinesia: a prospective qualitative study of knowledge sharing, symptom concealment, embarrassment, mistrust, and stigma. BMC Pulm Med 2006; 6: 25.

23 Carotenuto M, Esposito M, Di Pasquale F, et al. Psychological, cognitive and maternal stress assessment in children with primary ciliary dyskinesia. World J Pediatr 2013; 9: 312-317.

24 Pifferi M, Bush A, Di Cicco M, et al. Health-related quality of life and unmet needs in patients with primary ciliary dyskinesia. Eur Respir J 2010; 35: 787-794.

25 Lucas JS, Behan L, Galvin AD, et al. A quality-of-life measure for adults with primary ciliary dyskinesia: QOLPCD. Eur Respir J 2015; 46: 375-383. 
Smith BA, Modi AC, Quittner AL, et al. Dep its effects on adherence to airway clearance. Pediatr Pulmonol 2010; 45: 756-763.

52 Quittner AL, Abbott J, Hussain S, et al. Integration of mental health screening and treatment into cystic fibrosis clinics: evaluation of initial implementation in 84 programs across the United States. Pediatr Pulmonol 2020; 5: 2995-3004. 